\title{
Topological structures in finite temperature QCD
}

\section{Rasmus Larsen*}

Brookhaven National Laboratory, Upton, NY, 11973

E-mail: rlarsen@bnl.gov

\section{Sayantan Sharma}

The Institute of Mathematical Sciences, Chennai, 600113

E-mail: sayantanseimsc.res.in

\section{Edward Shuryak}

Stony Brook University, Stony Brook, NY, 11794

E-mail: edward. shuryakestonybrook. edu

\begin{abstract}
We report our study on the properties of the topological structures present in the QCD medium around the critical temperature $T_{c}$. We use dynamical domain wall fermion configurations on lattices of size $32^{3} \times 8$ to detect the topological structures through the zero and near-zero modes of the overlap operator. We show that the properties of the zero modes of the QCD Dirac operator agree well with that of calorons with non-trivial holonomy. We also show how the zero modes move around when changing the fermionic boundary conditions, and how the locality of the solutions also depends on the boundary conditions. This supports the presence of instantondyons in the hot QCD medium around $T_{c}$, where the distance between dyons control the shape and extent of the zero modes.
\end{abstract}

The 36th Annual International Symposium on Lattice Field Theory - LATTICE2018

22-28 July, 2018

Michigan State University, East Lansing, Michigan, USA.

${ }^{*}$ Speaker. 


\section{Introduction}

The aim of this work is to identify the topological objects that exist in the vacuum of QCD with physical quarks near the chiral crossover transition. Topological objects and their interactions play an important role in describing many features of chiral symmetry breaking and is believed to provide a possible mechanism for explaining confinement in gauge theories. The topological objects of interest in this present work are calorons with non-trivial holonomy. The caloron for the $S U$ (3) color group has been shown to consist of three substructures called instanton-dyons (dyons for short) [1]. For details of application of a model of instanton-dyons towards explaining confinement see [2]. Near the crossover temperature $T_{c} \sim 155 \mathrm{MeV}$ the Polyakov loop has a finite expectation value or non-trivial holonomy, which means that all three types of instanton-dyons are expected to exist. These would eventually recombine into instantons at $T>>T_{c}$. We will verify if indeed this is the scenario near $T_{c}$.

The topological objects in QCD always have a corresponding fermion zero mode through the index theorem. An ensemble of topological objects will interact to create near-zero modes with very small eigenvalues in addition to the exact zero modes. We will use the fermion zero and near-zero modes to identify the nature and the interactions of the topological objects. This is an indirect method, but it has the advantage that these modes are not sensitive to the high momentum fluctuations of the gauge fields. When well separated, each dyon and the associated fermion zero mode look like a localized peak. Each dyon correspond to a sector on the $U(1)$ circle between two successive angles $\mu_{i}$, as can be seen in Fig. 1, where $\mu_{i}$ are the angles of the expectation value of the Polyakov loop $\sim \exp \left[i \times \operatorname{diag}\left(\mu_{1}, \mu_{2}, \mu_{3}\right)\right]$. The fermion zero mode can only sit on one of the three dyons. Which dyon the zero mode sits on, depends on the boundary phase given by $\phi$ $\psi(t+1 / T)=e^{i \phi} \psi(t)$. The i'th dyon is associated with the zero mode if $\mu_{i}<\phi<\mu_{i+1}$. Close to $T_{c}$ we expect $\mu_{i} \sim 0,2 \pi / 3$ and $4 \pi / 3$. In order to detect all three dyons we therefore choose the phases $\phi=\pi, \pi / 3$ and $-\pi / 3$ such that they are at the center of the intervals in the phase circle.

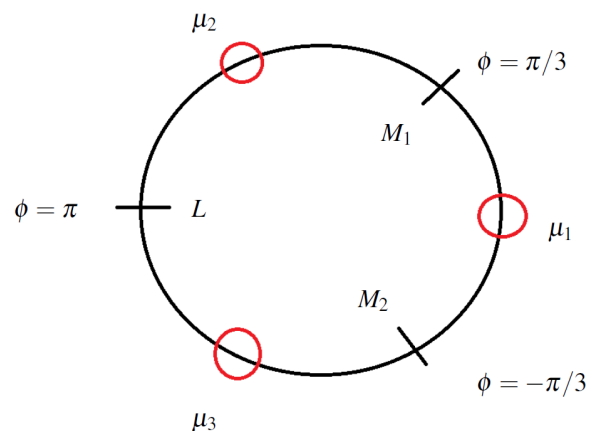

Figure 1: The different angles $\mu_{i}$ that governs the behavior of a caloron with non-trivial holonomy are shown along with the 3 different boundary phases $\phi$ studied in this work. The $\mu_{i}$ have been positioned such that $\langle P\rangle=0$. The 3 dyons depending on their location in the phase circle are called $L, M_{1}$ and $M_{2}$ respectively.

In order to study the topological structure of QCD with physical quarks, we use the $2+1$ flavor QCD configurations generated by the RBC-LLNL collaboration using Möbious domain wall discretization for fermions. The configurations have previously been used in [3]. The lattice size 
is $32^{3} \times 8$ and the quark masses are chosen at the physical values. We use the massless overlap Dirac operator with different boundary phases $\phi=\pi, \pi / 3$ and $-\pi / 3$ and calculate its first five eigenvalues and eigenvectors $\psi$ using the Kalkreuter Simma Ritz algorithm [4]. We use the overlap operator since it has an exact index even on a finite lattice. The quantities of interest are the eigen density $\rho(x)=\psi(x)_{a, i}^{\dagger} \psi_{a, i}(x)$ and chiral density $\rho_{5}(x)=\psi(x)_{a, i}^{\dagger} \gamma_{5(i, j)} \psi_{a, j}(x)$. Comparing the exact and near-zero modes to the analytic formula for the fermion zero mode for the caloron with non trivial holonomy $[1,5]$ we will verify if these indeed correspond to dyons.

\section{Our results: Exact zero modes}

We begin by comparing the spacetime profile of the density of a fermion zero mode on a typical QCD configuration at $T=1.08 T_{c}$ to the analytic results for a zero mode for confined case corresponding to a dyon and for a deconfined case corresponding to an instanton. The comparison is shown in fig. 2. We observe that the height of the dominant peak of the lattice zero mode is similar to the analytically calculated profile in the confined case for the boundary phase $\phi=\pi$.
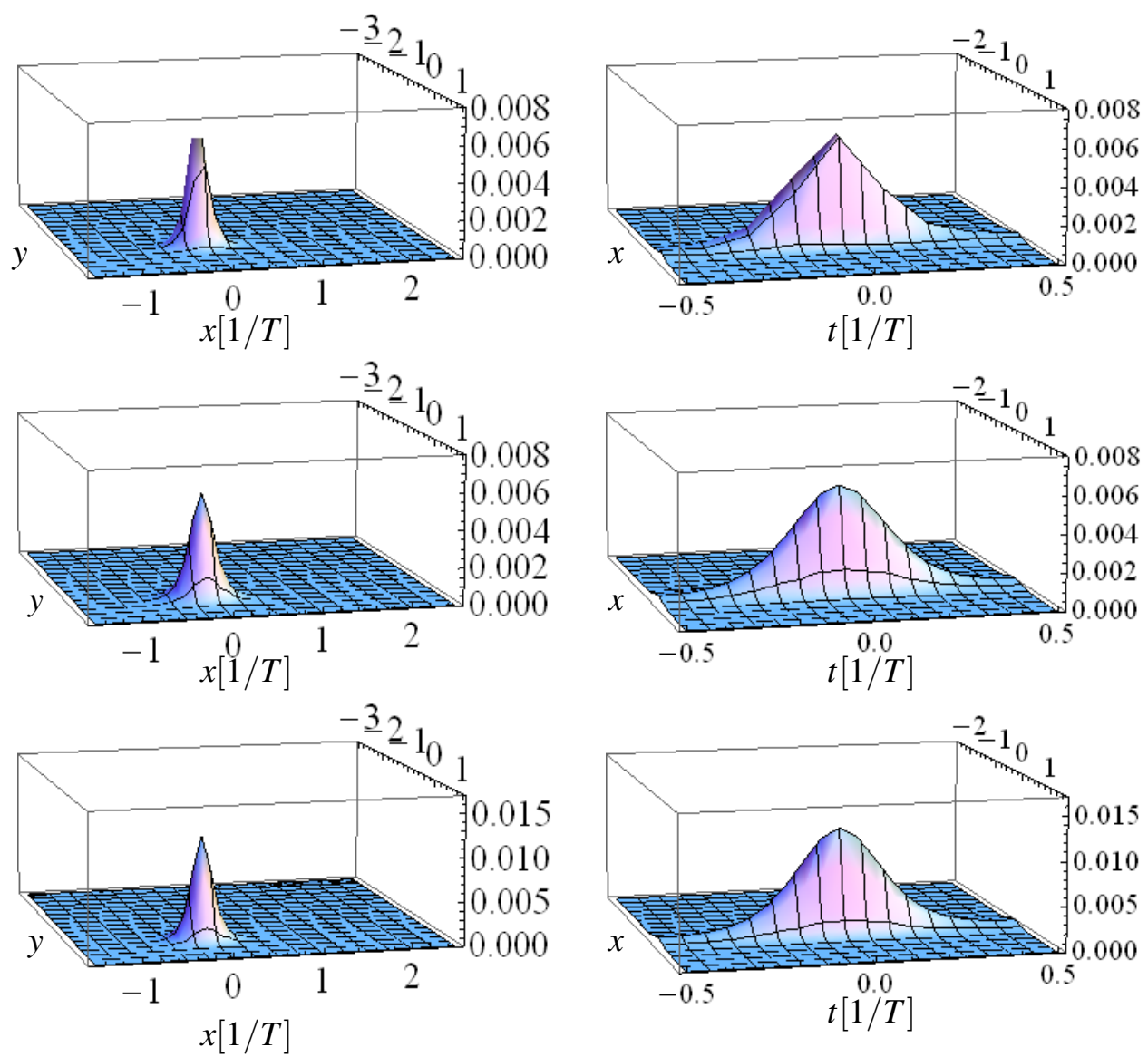

Figure 2: Fermion zero-mode densities $\rho(x, y)$ (left) and $\rho(x, t)$ (right) calculated from the lattice study (top panel) compared to the profile corresponding to the confined caloron (middle panel) and deconfined case (bottom panel). The dominant dyon in the analytic calculation is localized at the origin while the other two dyons sit at $(0.14,0,0) / T$ and $(-0.14,0,0) / T$. The axes in the plots are in units of $1 / T$. 


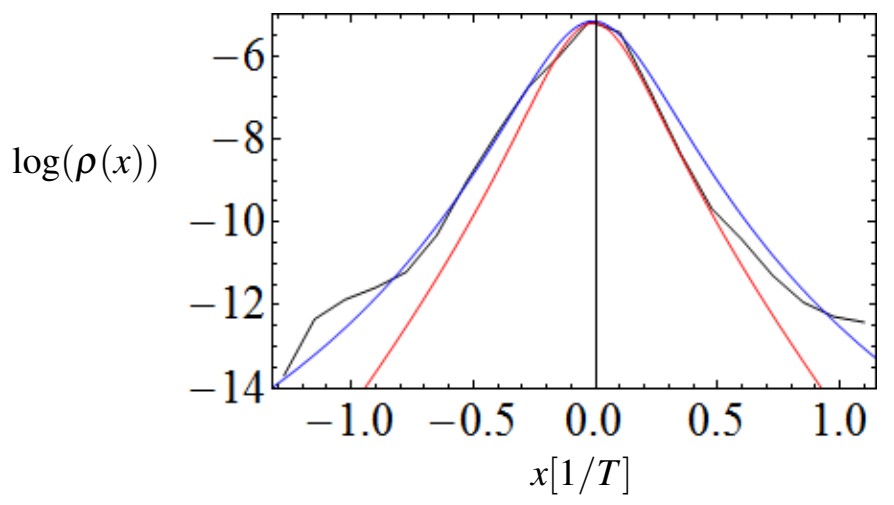

Figure 3: $\log (\rho(x))$ along the coordinate $x$ for the fermion zero mode (black) obtained from lattice simulation is compared with the analytically calculated caloron for confined (blue) and deconfined (Instanton) (red). For the analytic calculation, the main dyon sits at the origin, while the other dyons are localized at $(0.14,0,0) / T$ and $(-0.14,0,0) / T$ respectively. The axes are normalized by temperature $T$ to fit the analytic formula. The profile shown as a red line is scaled by a constant to fit the two other peak heights.

The profile plots of the same zero modes now in the $x$-t plane in Fig. 2 contain more interesting information. If the three dyons are well separated, then the solution is time independent. The closer the dyons are to each other, the more time dependent the solution becomes. The time dependence is therefore a good indication for the distance between the dyons. In order to further distinguish between the zero-mode profiles corresponding to a dyon and an instanton and compare with our lattice results, we show the density of this mode as a function of one of the space dimensions i.e. along the $\mathrm{x}$-direction as a log-plot in Fig. 3. We find that the peak corresponding to an instanton (red line) has a faster falloff as a function of $x$. This is due to the fact that the falloff of the zero mode solution depends on the distance from $\phi$ to the closest $\mu_{i}$, which is largest for the case of an instanton at $\phi=\pi$. Also the lattice result is not mirror symmetric along the $\mathrm{x}$-axis, which indicates that the other dyons are sitting close to one side. The plot also indicate that the actual value of the Polyakov loop is between 0 and 1 , and not at one of the two extremes, as assumed in all these plots.

\subsection{Comparing zero modes at different boundary phases}

We here show the lattice fermion zero mode profiles at $1.08 T_{c}$ as a function $x-y$ at a fixed $z$ coordinate and summing over the temporal direction, for different boundary phases $\phi=\pi, \pi / 3$ and $-\pi / 3$ summarized in Fig. 4 . We observe that for all three choices of $\phi$, one peak exists at the spacetime location where the anti-periodic case $(\phi=\pi)$ has a large peak. Due to several peaks contributing at $\phi=\pi / 3$ and $-\pi / 3$, we see that the peak height is much smaller than for the case $\phi=\pi$. We also observe that there are cases where the zero mode at a particular location survive for only one of the boundary phases as observed in the two plots corresponding to $z=19$ in Fig. 4 . This implies that the position of the zero-mode peak changes with the temporal boundary condition. This cannot happen if the zero mode is associated with an instanton with trivial holonomy. Instead this observation indicates that the zero mode is moving from one dyon to another. 

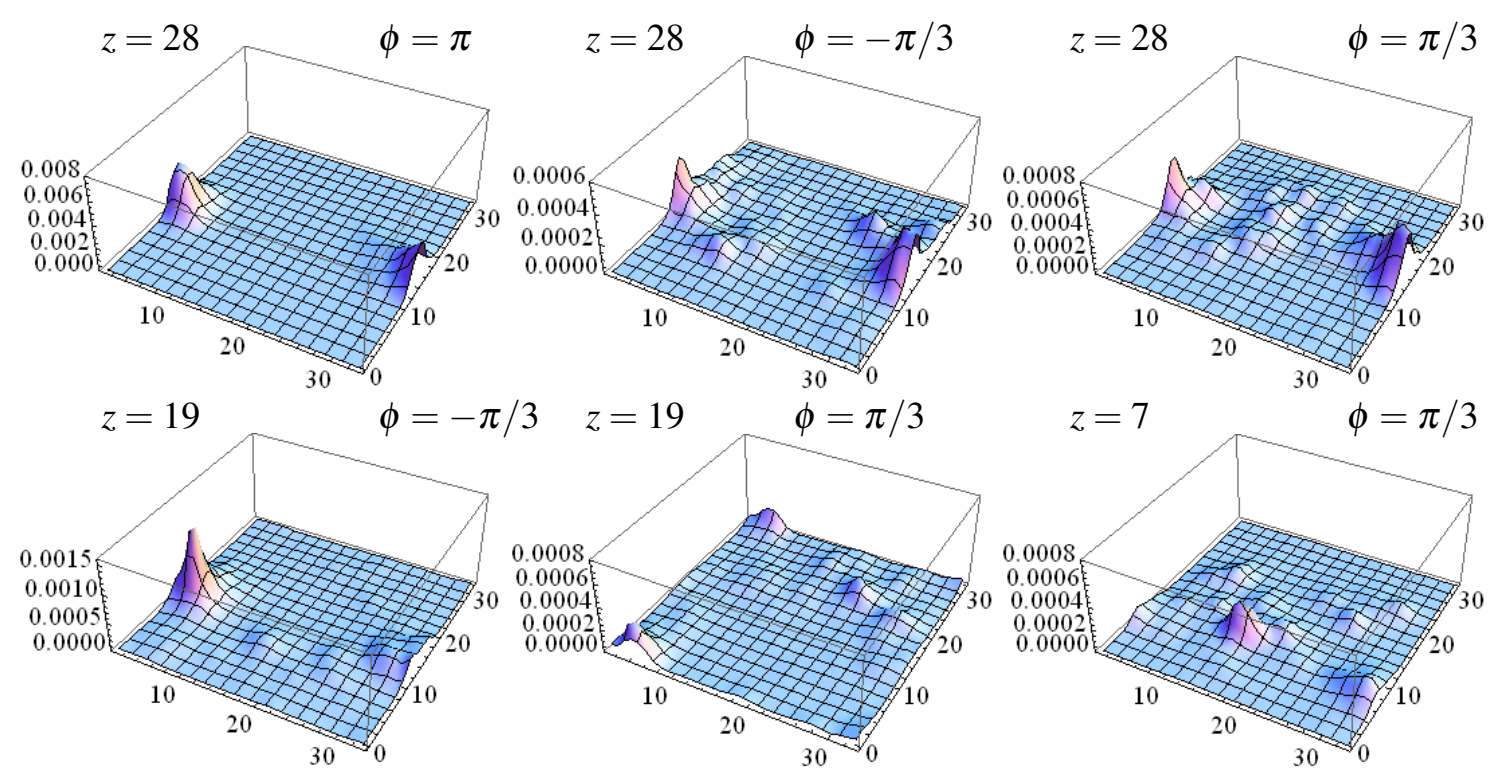

Figure 4: Fermion zero mode density $\sum_{t} \rho(x, y, t)$ at $T=1.08 T_{c}$. For (left top) $\phi=\pi, z=28$, (middle top) $\phi=-\pi / 3, z=28$, (right top) $\phi=\pi / 3, z=28$, (left bottom) $\phi=-\pi / 3, z=19$, (middle bottom) $\phi=\pi / 3, z=19$, (right bottom) $\phi=\pi / 3, z=7$. The axes are the lattice site along the respective directions.

\section{Near-Zero Modes of the QCD Dirac operator}

The near-zero modes arise due to the interactions between neighboring topological objects and their CP-odd partners. This can be seen in the chiral density profiles of the near-zero modes. We show the profiles for the first near-zero mode of the QCD Dirac operator for two different temporal boundary conditions in Fig. 5 at two different temperatures $T=1.08 T_{c}$ and $T \sim T_{c}$. We find that only above $T_{c}$ do we see very well separated peaks corresponding to positive and negative chiralities, while at $T_{c}$ we see a large density of peaks of both chiralities close to each other. The large density of peaks is consistent with chiral symmetry breaking scenario at $T_{c}$ and gives further evidence for the existence of dyons explained in detail in [6]. We give here a brief interpretation of our results. The QCD configurations were generated with anti-periodic boundary condition for the quarks, hence dyons only in the sector $\phi=\pi$ sector have been affected by fermionic zero mode attraction. This means that only the anti-periodic sector has a phase transition for chiral symmetry. This is not the case with the choice of other boundary phases corresponding to other dyons, which are not affected by the zero mode attraction. The plots therefore indicate that the fermion near-zero modes at the phases $\phi=\pi / 3$ and $-\pi / 3$, are associated with different topological object than the fermion near-zero modes in the anti-periodic sector $(\phi=\pi)$.

Even though near-zero eigenvectors correspond to several closely located peaks, it is still possible to get some nice comparisons between the analytic model and the closely located dyon-antidyon pairs. We show an example of this in Fig. 6 for the densities $\rho(x, y)$ and in fig. 7 for $\rho(x, t)$ for a typical configuration at $T=T_{c}$. 

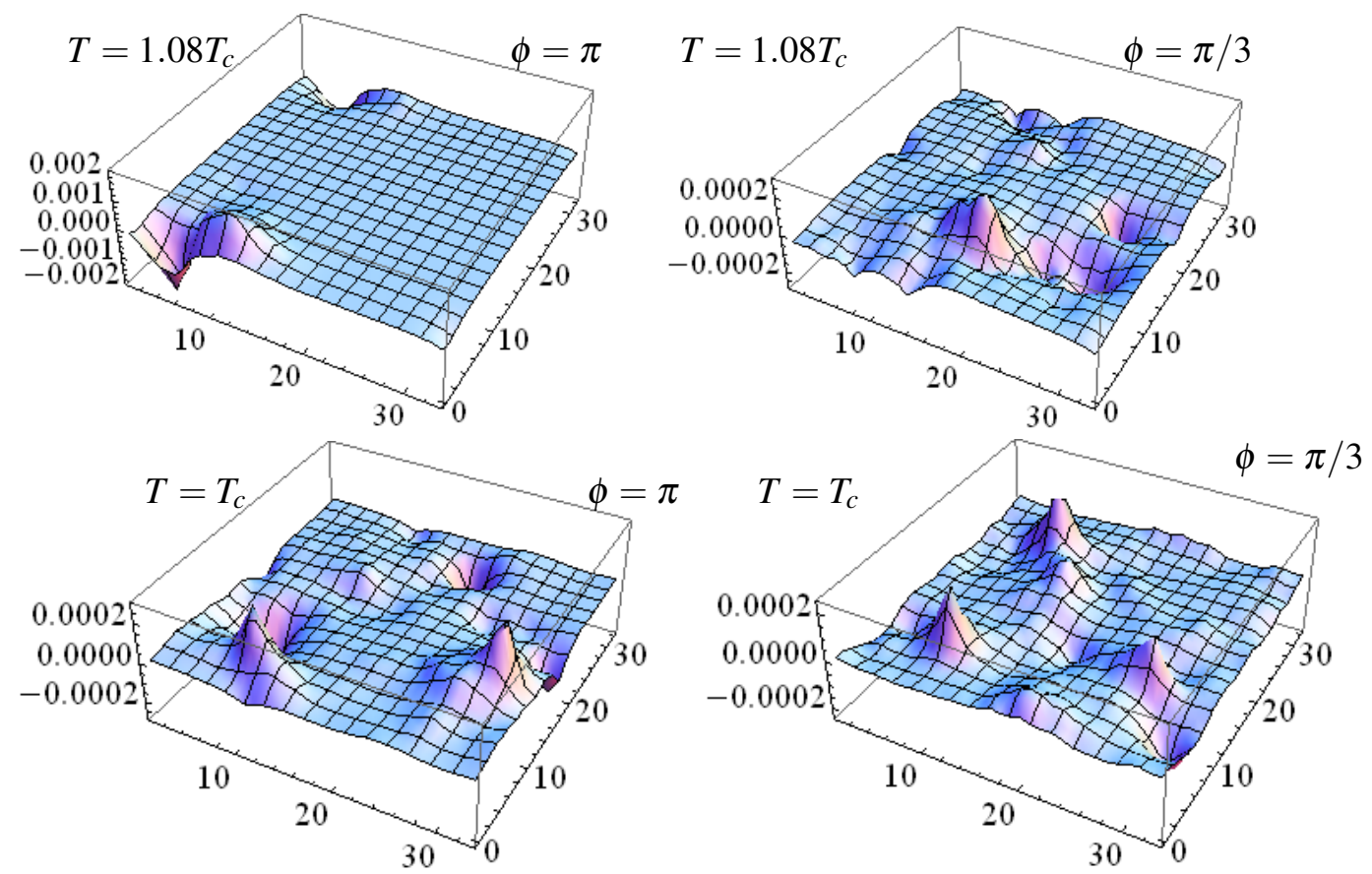

Figure 5: Chiral density $\sum_{t} \rho_{5}(x, y, t)$ at certain fixed $z$ plane of the first near-zero mode on the lattice at $T=1.08 T_{c}$ (top) and $T=T_{c}$ (bottom) as a function of fermion boundary phases $\phi=\pi$ (left) and $\phi=\pi / 3$ (right) respectively. The axes are the lattice site along the respective spatial directions.
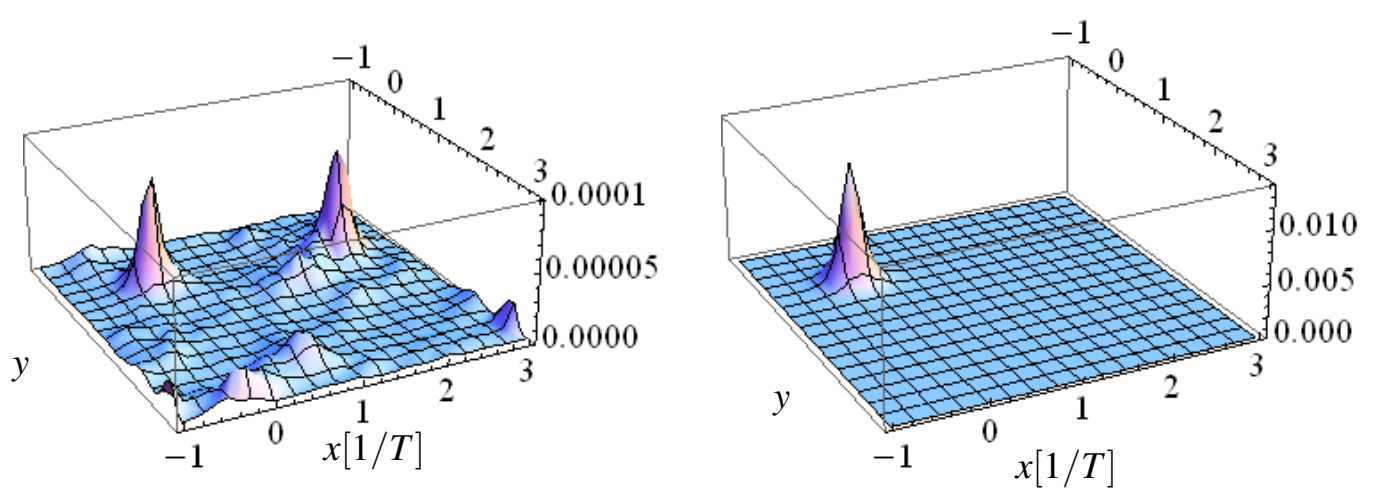

Figure 6: Near-zero mode density $\rho(x, y)$ at $T=T_{c}$ and $\phi=\pi / 3$ from lattice (left) and analytic (right) calculations. Lattice units are normalized by temperature $T$ to fit to the analytic result.

\section{Summary}

In this work we show that the fermion zero and near-zero modes of the QCD Dirac operator calculated on the lattice near and above $T_{c}$ correspond very well to the zero modes of the caloron with non-trivial holonomy i.e. dyons. We provide evidences for it through direct comparison of the shape of the peaks between lattice and analytic results as the boundary phases of the overlap operator is changed. Looking at the chiral density, only above $T_{c}$ at $\phi=\pi$ could we find configurations which were composed of a well separated single dyon and anti-dyon pair. At the other boundary phases studied, corresponding to $\phi=\pi / 3$ and $-\pi / 3$ above $T_{c}$, and all phases at $T_{c}$, we 

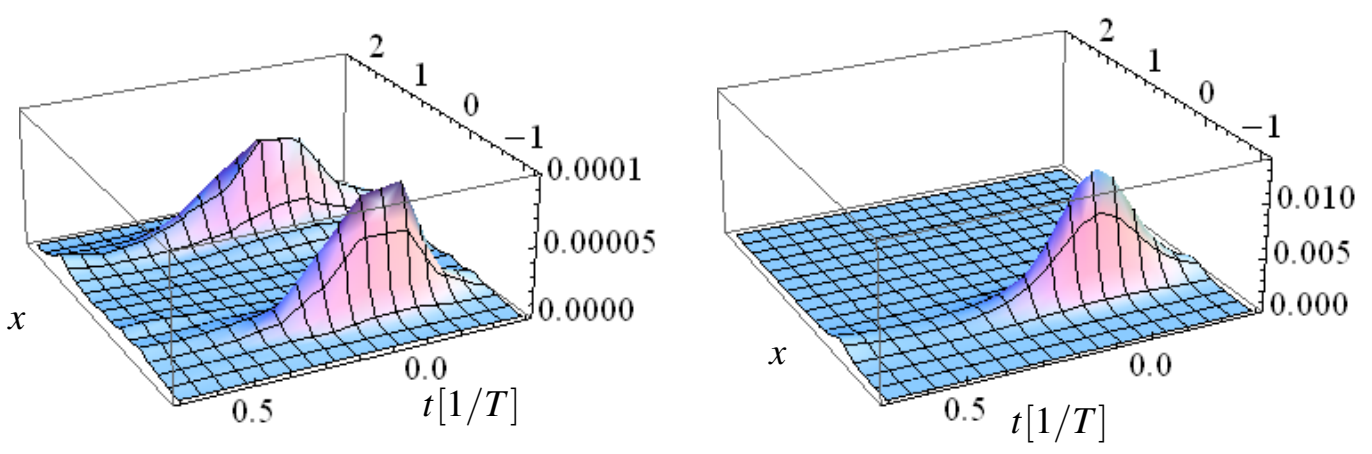

Figure 7: Near-zero mode density $\rho(x, t)$ at $T=T_{c}$ and $\phi=\pi / 3$ calculated on lattice (left) and analytic (right) calculations. Lattice units normalized by temperature $T$ to fit to the analytic result.

found a high density of peaks and valleys. This shows that only for $\phi=\pi$ was there a chiral phase transition, which in turn means that the topological objects in the two other sectors are different from that in the anti-periodic case. We also saw that while one large peak tended to be close to the same position of the exact zero mode for $\phi=\pi$, we did also see peaks shift to different locations for the other two boundary phases. This shows that the topological objects move around for different boundary conditions, another strong indication that the vacuum is composed of dyons. All of these results support the presence of instanton-dyons in the hot QCD medium around $T_{c}$.

Though this method is an indirect method where the presence and nature of topological objects are studied using the fermion zero modes, we have shown that it is a clean technique which can be used to identify dyons in QCD, as was also shown earlier in pure gauge theory [7].

Acknowledgements: This work was supported in part by the Office of Science, U.S. Department of Energy, under Contract Nos. DE-FG-88ER40388 (E.S.) and DE-SC001270 (R.L.). The computations were performed with the GPU cluster at the Institute of Mathematical Sciences. We thank P. Mangalapandi for technical support. Our GPU code is in part based on some publicly available QUDA libraries [8].

\section{References}

[1] T. C. Kraan and P. van Baal, Nucl. Phys. B 533, 627 (1998)

[2] D. Diakonov, Nucl. Phys. Proc. Suppl. 195, 5 (2009)

[3] T. Bhattacharya et al., Phys. Rev. Lett. 113, no. 8, 082001 (2014)

[4] T. Kalkreuter and H. Simma, Comput. Phys. Commun. 93, 33 (1996).

[5] M. N. Chernodub, T. C. Kraan and P. van Baal, Nucl. Phys. Proc. Suppl. 83, 556 (2000)

[6] R. Larsen, S. Sharma and E. Shuryak, in preparation.

[7] C. Gattringer, Phys. Rev. D 67, 034507 (2003)

[8] M. A. Clark et. al., Comput. Phys. Commun. 181, 1517 (2010). 